\title{
A new hybrid algorithm for solving distribution network reconfiguration under different load conditions
}

\author{
Omar Muhammed Neda \\ Depertmant of Engineering Affairs, Sunni Diwan Endowment, Iraq
}

\begin{tabular}{l}
\hline \hline Article Info \\
\hline Article history: \\
Received Dec 2, 2019 \\
Revised May 11, 2020 \\
Accepted Jun 6, 2020 \\
\hline
\end{tabular}

\section{Keywords:}

BPSO

CBPSO

DNR

MATLAB

Power loss

Voltage profile

\begin{abstract}
Distribution Network Reconfiguration (DNR) is a significant problem for keeping the network running under normal conditions. In this study, for preventing the premature convergence issue, also to improving the searching ability of the Binary Particle Swarm Optimization (BPSO) algorithm, chaotic strategy is incorporating with BPSO algorithm to create a new hybrid algorithm called Chaotic BPSO (CBPSO). Undeniably, the chaotic strategy enables the hybrid CBPSO algorithm to slip from the local optima and also to reach optimal solution in fewer number of iterations compare to BPSO due to the remarkable behavior and ergodic of the chaos strategy than random search in BPSO algorithm. The CBPSO algorithm is presented as a advantageous optimization tool for solving DNR. In this problem, decreasing of real power loss $\left(\mathrm{P}_{\mathrm{L}}\right)$ is an objective function while node voltage, system radially and line current have been utilized as a constrains of the system. The search space in this problem for the presented technique is a group of lines (switches) that are normally opened or closed. Two types of loads are presented: the constant and variable loads for testing the efficacy of the CBPSO method for tackling DNR problem when the load is changes. The proposed technique is implemented on IEEE Node-33 system by utilizing MATLAB R2013b software for verifying the efficacy of CBPSO technique. The simulation results confirm that CBPSO technique has high ability in reducing $\mathrm{P}_{\mathrm{L}}$ and raising the voltage profile of the grid compared to BPSO and other procedures in the literature.
\end{abstract}

Copyright $(2020$ Institute of Advanced Engineering and Science. All rights reserved.

\section{Corresponding Author:}

Omar Muhammed Neda,

Department of Electrical Power Engineering,

Sunni Diwan Endowment, Bagdad, Iraq.

Email: omar.neda88@gmail.com

\section{INTRODUCTION}

Loss minimization is used so as to improve the flexibility and efficacy of the system. Distributed Generator (DG) allocation, conductor grading, capacitor placement and feeder reconfiguration are better approaches for decreasing power loss [1]. On the other hand, adding these methods into the distribution system needs much cost. In this work, feeder reconfiguration is presented to decrease power loss and keep the voltage at all nodes with an acceptable limit. DNR can be attained through the reconfiguration of tie switches and sectionalizing, by this method, the loss system is reduced and voltage level is enhanced by considering the operating limits devoid of costs [2]. By redistributing and arranging the loads from heavy to light, DNR can balance the feeder loads in addition to prevents the overloading [3].

Numerous techniques are described in the literature to obtain the optimal DNR. Artificial Neural Network (ANN) technique is presented using the mapping ability to determine the reconfiguration of the grid [4]. An expert system utilizing heuristic rules for reducing the search problem for decreasing the calculation time has been proposed in [5]. The study of load balancing and reducing power loss formulated as integer programming problem was proposed by Baran [6]. Chiang and Jumeau have been presented a new load 
balancing index as well as they utilized it on the test power system to load balance [7]. A new balance and unbalance load approach in distribution system for decreasing of the power loss was presented in reference [8]. Naveen was presented DNR for reducing loss via modification technique based on the bacterial foraging optimization [9]. Cuckoo Search Approach (CSA) was introduced by Nguyen and Truong; DNR have two goals, which were to voltage level enhancement as well as to diminish the loss of the system [10].

The branch exchange technique is presented in [11]. In this technique, power loss minimization is achieved. A technique was presented to achieve switch configurations as a function of time [12]. Oliveira et al. proposed a technique for the goal of reducing energy loss by utilizing Artificial Immune (AI) and presented on Node-33 system [13]. An application utilizing Genetic Algorithm (GA) was presented in [14]. Improved GA (IGA) based on fuzzy multi-objective method was proposed for solving this problem [15]. Ant Colony Search Algorithm (ACSA) was offered for solving the DNR problem. Furthermore, they have achieved a comparison with Simulated Annealing (SA) and GA which displays superior implications for ACSA [16]. A solution method by utilizing SA is presented to search for an satisfactory noninferior solution [17-18]. Harmony Search Algorithm (HSA) is presented for solving DNR problem as well as an optimum grid switching combination is attained with implications in lowest loss [19]. Jin et al. have presented Binary PSO (BPSO) method for balancing the loads of the network [20].

Between all these algorithms, BPSO has greatly effective in obtaining global solution and shown reliability to prevent these disadvantages of the traditional algorithms that reported in the literature and can easily applied to multi problems but it does not mean that BPSO algorithm not involves any drawbacks. However, in solving non-linear problem this algorithm is declining very easily to the local minima on the other hand also it is relying on its parameters settings. So, the researchers working to enhance and develop PSO algorithm to avoids these disadvantages by using several techniques combined with BPSO algorithm.

In this work, so as to enhance the convergence characteristic, performance and to inhibit the problem of the premature convergence, which occurs when the algorithm is trapped into a local optimum through the search process in the BPSO technique, a chaotic strategy is merged with BPSO technique to form a new hybrid algorithm called Chaotic BPSO (CBPSO). This blending very much helps the hybrid CBPSO algorithm to slip more easily from the local optima due to the remarkable behavior, dynamic properties and ergodic of chaos strategy than random search in BPSO approach. In the presented work, BPSO and CBPSO techniques are presented as an optimization tools for the solution of DNR issue, for decreasing the power loss and improving voltage profile of the distribution system. The BPSO and CBPSO algorithms are applied on IEEE Node -33 system with constant loads and variable loads in order to examining and evaluating the ability and reliability of these algorithms (i.e. BPSO and CBPSO algorithms) to solve this problem at any change in loads and to find the optimal DNR. For variable load ( $\mu$ multiplied by constant load) where $\mu$ denotes the ratio value for the load deviation. The range of variation for loads is linearly changed between $(\mu=0.75)$ at light load up to $(\mu=1.250)$ at heavy load. The simulation results emphasize that CBPSO algorithm has high ability and effective in reducing the power loss as well as enhancing minimum and average voltages of the system compared to BPSO and other algorithms in the literature.

\section{PROBLEM FORMULATION}

\subsection{Load Flow}

In this work, the load flow calculations are automatically achieved by utilizing MATPOWER when running the program. Load flow in distribution system can be defined through a number of equations that depends on the active, reactive power in addition to voltage at the sending end of a line for expressing the same quantities at receiving end of the line [21]. By utilizing the calculation of power flow, power loss can be obtained as revealed in Figure 1.

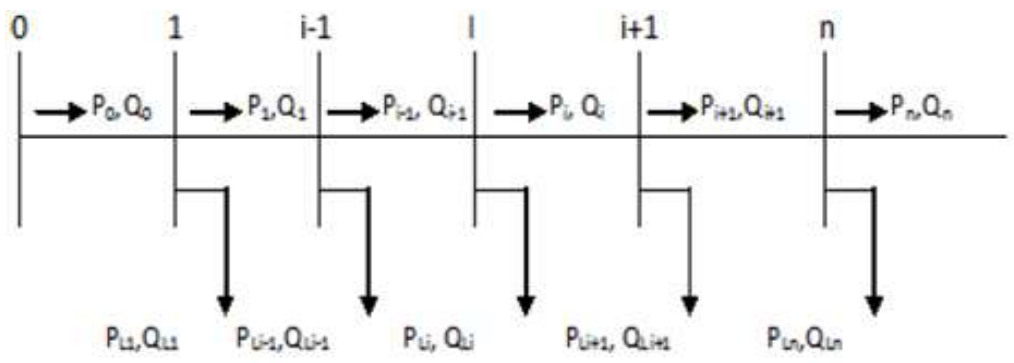

Figure 1. Simple distribution line 
The active as well as reactive load flow equations in the branch among $i+1$ and $n-t h$ nodes are:

$$
\begin{aligned}
& P_{i+1}=\left[\left(P_{i}\right)-\left(P_{L i+1}\right)-\left(R_{i, i+1}\right)\right]\left[\frac{\left(P_{i}\right)^{2}+\left(P Q_{i}\right)^{2}}{\left|V_{i}{ }^{2}\right|}\right] \\
& Q_{i+1}=\left[\left(Q_{i}\right)-\left(Q_{L i+1}\right)-\left(X_{i, i+1}\right)\right]\left[\frac{\left(P_{i}\right)^{2}+\left(Q_{i}\right)^{2}}{\left|V_{i}{ }^{2}\right|}\right]
\end{aligned}
$$

The voltage at nodes $i$ and $i+1$ can be express as follows:

$$
\left|\left(V_{i+1}\right)^{2}\right|=\left|\left(V_{i}\right)^{2}\right|-2\left[\left(R_{i, i+1} \cdot P_{i}\right)+\left(X_{i, i+1} \cdot Q_{i}\right)\right]+\left[\left(R_{i, i+1}^{2}\right)+\left(X_{i, i+1}^{2}\right)\right]\left[\frac{P_{i}{ }^{2}+Q_{i}{ }^{2}}{\left|V_{i}{ }^{2}\right|}\right]
$$

The current can be express by Eq. (4):

$$
I_{T}=\frac{P_{i}-j Q_{i}}{\left|V_{i}\right|}
$$

The summation of actual power loss can be express as shown below:

$$
P_{L T}=\sum R_{i, i+1} * I_{T}^{2}
$$

From the above equations: $\left(P_{i}\right),\left(Q_{i}\right)$ : The actual and reactive power; $\left(R_{i, i+1}\right),\left(X_{i, i+1}\right)$ : The resistance in addition to reactance of branch section among two nodes $i$ and $i+1 ;\left(V_{i}\right)$ : The voltage at node $i ;\left(I_{T}\right)$ : depicts the total current and $\left(P_{L T}\right)$ : The total real power losses.

\subsection{Objective Function}

The great target of DNR is for decreasing the actual power loss and it is presented in (6):

$$
f(x)=\min P_{L T}
$$

where $(x)$ is the control variable and $P_{L T}$ is the total power loss.

\subsection{Constrains}

The load flow calculation can be done by finding the node voltage, line current in addition to active power loss of a system of each line. The necessities of the objective function are shown below:

1. Bus voltage has min and $\max$ bounds as shown in (7).

$$
V_{i}^{\min } \leq\left|V_{i}\right| \leq V_{i}^{\max } ; i=1,2, \ldots . . N_{n}
$$

From equation (6), $V_{i}^{\min }$ and $V_{i}^{\max }$ are the lower (0.9 p.u min) and upper (1.0 p.u max) voltage of node $i ; N_{n}$ represent the number of nodes.

2. Line current values should not overcome constraint of each line as in (8).

$$
\left|I_{T}\right| \leq I_{T}^{\max } ; T=1,2, \ldots . N_{b}
$$

Where $I_{T}{ }^{\max }$ is the $\max$ bound of line current $T$ and $N_{b}$ depicts the whole number of the lines.

3. Always save the power system in radial structure as written in (9).

$$
\left.\begin{array}{l}
\operatorname{det}(A)=1 \text { or }-1(\text { Radial System }) \\
\operatorname{det}(A)=0(\text { Not Radial System })
\end{array}\right\}
$$

\section{OPTIMIZATION PROCESS}

\subsection{BPSO Algorithm}

PSO is one of the effective and efficient optimization techniques [22]. Basic idea of PSO came from the random behavior of animals like bird flocking [23-24]. The basic PSO algorithm is the real valued, whereby every dimension in the space of the problem can take any real valued number. The first concept for 
BPSO algorithm has been presented by Eberhart and Kennedy in year 1997 [25]. So as to transform the exploration of PSO in a real space dimensions to binary space dimensions, sigmoid transformation is applied to the velocity element to force the velocities within $[0,1]$, and force the component values of the positions of agents to be ( $0 \mathrm{~s}$ or $1 \mathrm{~s})$. So, the equations of BPSO are:

$$
\begin{aligned}
& v_{i}{ }^{k+1}=\left(W_{B P S O} * v_{i}{ }^{k}\right)+\left[c_{1} * \operatorname{rand}_{1} *\left(p_{b i}{ }^{k}-x_{i}{ }^{k}\right)\right]+\left[c_{2} * \operatorname{rand}_{2} *\left(g_{b i}{ }^{k}-x_{i}{ }^{k}\right)\right] \\
& \operatorname{sigmoid}\left(v_{i}^{k+1}\right)=\frac{1}{1+\exp \left(-v_{i}^{k+1}\right)} \\
& \left.x_{i}^{k+1}=\left[1, \text { if rand }<\operatorname{sigmoid}\left(v_{i}^{k+1}\right)\right]\right\} \\
& x_{i}^{k+1}=[0, \text { otherwise }]
\end{aligned}
$$

From the above equations, $\left(v_{i}{ }^{k+1}\right)$ is the velocity of individual; $\left(c_{1}, c_{2}\right)$ are the two positive constants within $[0-2.5] ;\left(\operatorname{rand}_{1}, \operatorname{rand}_{2}\right)$ are the random numbers within bound [0-1]; $\left(p_{b i}{ }^{k}\right)$ is the local best value; $\left(g_{b i}{ }^{k}\right)$ is the global best value; $\left(x_{i}^{k}\right)$ is the position; and $\left(W_{B P S O}\right)$ : is the inertia weight and it is reduce linearly from ( 0.9 to 0.4$)$ at each iteration, and it can be express as follows [26-27]:

$$
W_{B P S O}=W_{\max }-\left(\frac{W_{\max }-W_{\min }}{\text { max }_{\text {iteration }}}\right) * \text { iter }
$$

From the above equation:

$W_{\min }, W_{\max }$ : The initial and final inertia weight.

iter: The present iteration.

$\max _{\text {iteration: }}$ The max. iterations.

\subsection{CBPSO Algorithm}

In spite of the advantages of BPSO algorithm but often it has some disadvantages similar to the other techniques, and the main disadvantages in BPSO algorithm are highly relies on its parameters and plunge to the local optima near optimal solution when the problem is very complex and contains very large numbers of variables. In order to prevents these disadvantages and to enhance the quality and convergence characteristic, a chaos strategy merged with a BPSO algorithm to form a hybrid algorithm called CBPSO and this way very helping the CBPSO to slip from the local optima due to the remarkable behaviour and high ability of the chaos strategy than random search in BPSO algorithm [28]. In this study, the logistic sequence equation adopted for establishing the hybrid CBPSO algorithm is described by the following equation [29]:

$$
\beta^{k+1}=\mu \beta^{k}\left(\left(1-\beta^{k}\right)\right), 0 \leq \beta^{1} \leq 1
$$

From equation (18), the control parameter $\mu$ is set within a range [0.0-4.0], $k$ is the number of the iterations. The magnitude of $\mu$ decides whether $\beta$ stabilizes at a constant area, fluctuates insides restricted limits, or operates chaotically in an unforeseeable manner. And (18) is deterministic, it shows chaotic dynamics when $\mu=4.0$ and $\beta^{1} \in\{0,0.25,0.5,0.75,1\}$. It displays the sensitive depend on its initial conditions, which is the basic features of chaos. The new weight factor $\left(W_{C B P S O}\right)$ is calculated by multiplying the $\left(W_{B P S O}\right)$ in (13) and logistic sequence in (14) to form (15):

$$
W_{C B P S O}=W_{B P S O} * \beta^{k+1}
$$

To enhance the behavior and the searching ability of the BPSO, this study presents a new velocity change by merging a logistic sequence equation $(\beta)$ with inertia weight factor $\left(W_{B P S O}\right)$. Finally, the following velocity updated equation for the suggested CBPSO is achieved as display in (16):

$$
v_{i}^{k+1}=W_{\text {CBPSO }} * v_{i}^{k}+C_{1} * r_{1} *\left(p_{\text {best }(i)}^{k}-x_{i}^{k}\right)+C_{2} * r_{2}^{*}\left(g_{\text {best }(i)}^{k}-x_{i}^{k}\right)
$$

In the CBPSO algorithm, $W_{C B P S O}$ is decrease and oscillates simultaneously from (0.9 to 0.4$)$ for total iteration but in BPSO is reducing linearly. And the particle update your position is the same as in BPSO algorithm. Figure 2 displays the flowchart for the CBPSO technique. 


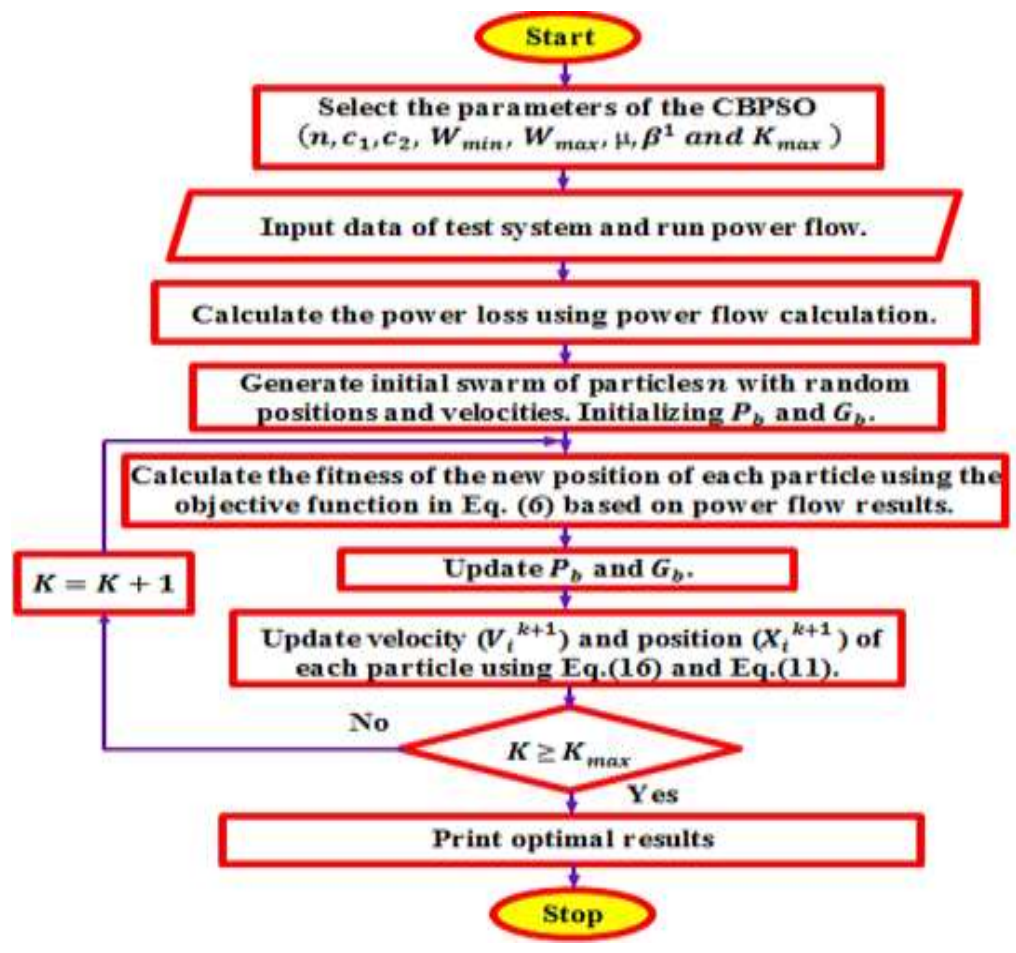

Figure 2. CBPSO algorithm for solving DNR problem

\section{CASE STUDY AND RESULTS}

The efficiency, usefulness and applicability of CBPSO for the solution of DNR is verified and tested on IEEE Node-33 system. The data details of the network and loads for power system have been given in [10]. And the results of the network reconfiguration at BPSO and CBPSO are obtained in two cases at constant load and at variable load.

\subsection{Case Study (1) with Constant Load}

In this case, DNR is implemented to the constant load $(\mu=1)$ demand. IEEE 33 node is presented as test system for both the BPSO and CBPSO approaches. Table 1 describes the comparison among the proposed methods and some other methods reported in the literature $[2,9,10,30]$. Switches status, $P_{L}$, minimum and average voltage are explained in this table.

Table 1. Numerical results of DNR for the IEEE 33-node at constant load demands $(\mu=1)$ while using BPSO, CBPSO and some other approaches

\begin{tabular}{ccccc}
\hline Approach & Open Switches & $P_{L T}(\mathrm{KW})$ & $V_{a v}(p . u)$. & $V_{\min }(p . u)$. \\
\hline Initial & sw33, sw34, sw35, sw36, sw37 & 202.67 & 0.9485 & 0.9092 \\
BPSO & sw7, sw9, sw13, sw32, sw37 & 138.61 & 0.9657 & 0.9412 \\
CBPSO & sw7, sw9, sw14, sw32, sw37 & 133.17 & 0.9669 & 0.9431 \\
FWA [2] & sw7, sw9, sw14, sw28, sw32 & 139.55 & 0.9674 & 0.9413 \\
MBFOA [9] & sw7, sw9, sw13, sw32, sw37 & 141.91 & 0.9678 & 0.9378 \\
ITS [10] & sw07, sw09, sw14, sw36, sw37 & 142.16 & 0.9653 & 0.9336 \\
SLR [30] & sw07, sw10, sw14, sw36, sw37 & 142.67 & 0.9651 & 0.9336 \\
\hline
\end{tabular}

Table 1 revels that the loss $\left(P_{L T}\right)$ reduces while using BPSO by $31 \%$ from $202.67 \mathrm{~kW}$ to $138.61 \mathrm{~kW}$ and with CBPSO by $34 \%$ from $202.67 \mathrm{~kW}$ to $133.17 \mathrm{~kW}$. The minimum voltage $\left(V_{\min }\right)$ while using BPSO enhances from 0.9092 to $0.9412 \mathrm{p}$. u. and with CBPSO enhances from 0.9092 to $0.9412 \mathrm{p} . \mathrm{u}$., while the average voltage enhances from 0.9485 to $0.9657 \mathrm{p}$. u. while using BPSO and from 0.9485 to 0.9669 p.u. with CBPSO.

Figure 3 shows voltage profiles of the network while using BPSO and CBPSO. It is clear that the voltage at all nodes (except 19, 20,21,22) were improves after reconfiguration. Finally, it is notice that from Figure 3 greatly improves the voltage profile by using CBPSO compared to BPSO. 

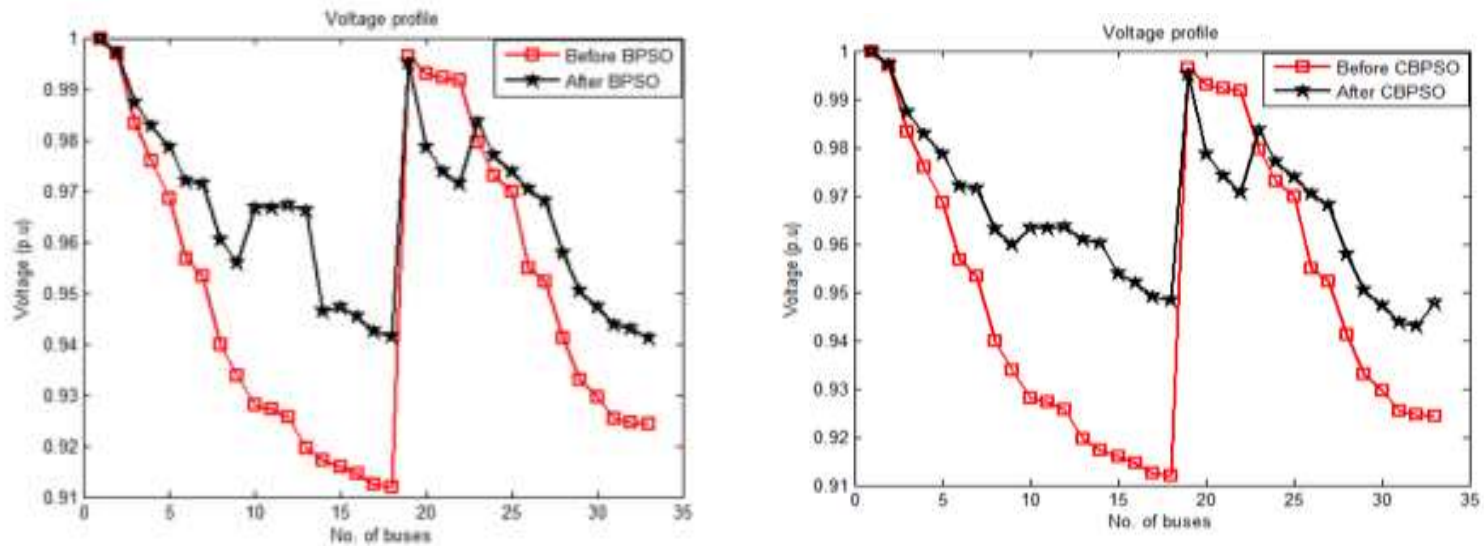

Figure 3. Voltage profile of DNR for the IEEE 33-node grid at constant load demands $(\mu=1)$ while using BPSO and CBPSO

\subsection{Case Study (2) With Variable Load}

This case is utilized to test and checked the ability, potential and efficiency of CBPSO algorithm for solving DNR problem at any change in load. The load demand (real and reactive) at all nodes is changes within the range $\left(\mu^{\min } \leq \mu \leq \mu^{\max }\right)$ where $\left(\mu^{\min }=0.75\right)$ at light and $\left(\mu^{\max }=1.25\right)$ at heavy loads with the percent of step change $(\Delta \mu)$ equal to $12.5 \%$. The load is varied by multiplying $\mu$ with load at base case.

$$
\begin{aligned}
& P_{L i}=\mu P_{L i 0} \\
& Q_{L i}=\mu Q_{L i 0}
\end{aligned}
$$

From the above equations:

$\mu$ : The magnitude of the load variation ratio.

$P_{L i 0}, Q_{L i 0}$ : The initial (active and reactive) powers at load nodes.

\subsubsection{At Loading with Variation Ratio $(\mu=0.75)$}

In this case DNR is implemented to the light load $(\mu=0.75)$ demand. IEEE 33 node is presented as test system for both the BPSO and CBPSO approaches. Table 2 describes the comparison among the proposed methods and some other methods reported in the literature $[2,9,10,30]$. Switches status, $P_{L}$, minimum and average voltage are tabulated in this table.

Table 2 reports that the loss $\left(P_{L T}\right)$ reduces while using BPSO by $30 \%$ from $109.75 \mathrm{~kW}$ to $76.16 \mathrm{~kW}$ and with CBPSO by $34 \%$ from $109.75 \mathrm{~kW}$ to $72.33 \mathrm{~kW}$. The minimum voltage $\left(V_{\min }\right)$ while using CBPSO enhances from 0.9362 to 0.9565 p.u. and with CBPSO enhances from $0.9362 \mathrm{p}$. u. to $0.9579 \mathrm{p} . \mathrm{u}$. while the average voltage enhances from 0.9621 to $0.9746 \mathrm{p}$.u. while utilizing BPSO and from 0.9621 to 0.9754 p. u. So, it is quite obvious from the comparison results that the CBPSO algorithm is achieved best results and it has high ability and superior in solving DNR problem than other methods.

Figure 4 shows voltage profiles of the network while using BPSO and CBPSO. It is clear that the voltage at all nodes (except 19,20,21,22) were improves after reconfiguration. Finally, it is clear that from Figure 4 by using CBPSO greatly improves the voltage profile compared to using BPSO.

Table 2. Results of DNR for the IEEE 33-node power system at light load demands $(\mu=0.75)$ while using BPSO, CBPSO and some other approaches

\begin{tabular}{ccccc}
\hline Approach & Open Switches & $P_{L T}(\mathrm{KW})$ & $V_{a v}(p . u)$. & $V_{\min }(p . u)$. \\
\hline Initial & sw33, sw34, sw35, sw36, sw37 & 109.75 & 0.9621 & 0.9362 \\
BPSO & sw7, sw9, sw13, sw32, sw37 & 76.16 & 0.9746 & 0.9565 \\
CBPSO & sw7, sw9, sw14, sw32, sw37 & 72.33 & 0.9754 & 0.9579 \\
FWA [2] & sw7, sw9, sw14, sw28, sw32 & 76.87 & 0.9758 & 0.9566 \\
MBFOA [9] & sw7, sw9, sw13, sw32, sw37 & 77.88 & 0.9762 & 0.9540 \\
ITS [10] & sw07, sw09, sw14, sw36, sw37 & 77.97 & 0.9743 & 0.9510 \\
SLR [30] & sw07, sw10, sw14, sw36, sw37 & 78.25 & 0.9742 & 0.9510 \\
\hline
\end{tabular}



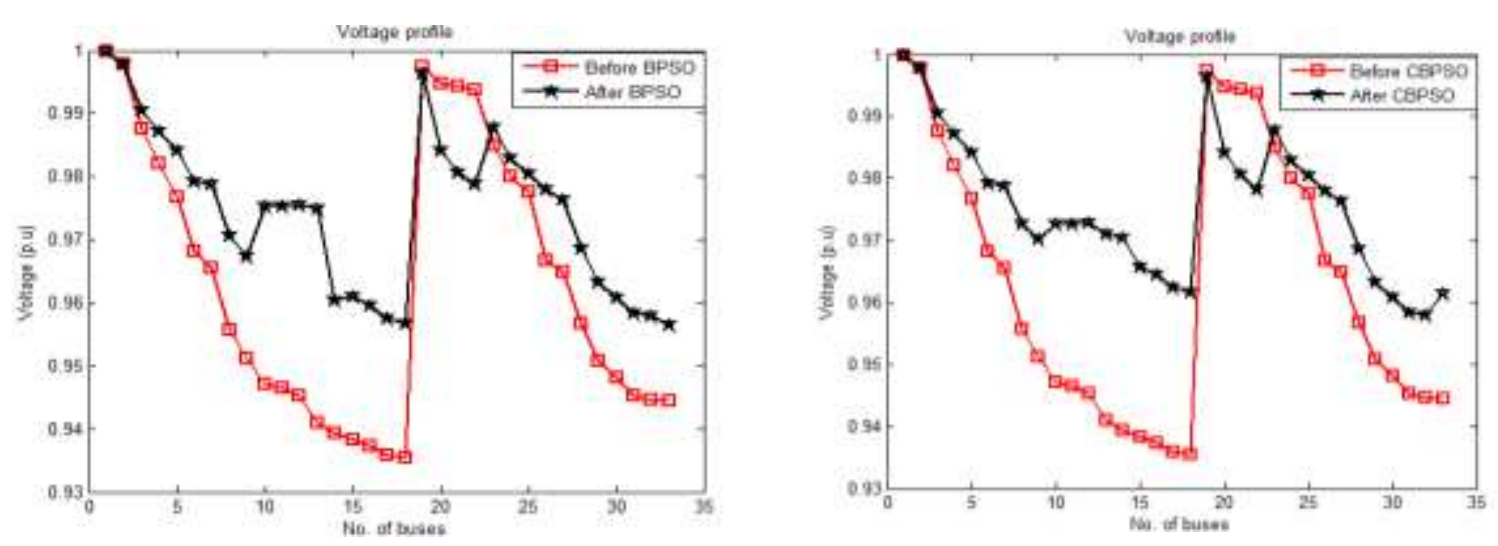

Figure 4. Voltage profile of DNR for the IEEE 33-node grid at light load demands $(\mu=0.75)$ while using BPSO and CBPSO

\subsubsection{At Loading with Variation Ratio $(\boldsymbol{\mu}=\mathbf{0 . 8 7 5})$}

In this case DNR is implemented to the load factor $(\mu=0.875)$ demand. IEEE 33-node is presented as test system for both the BPSO and CBPSO approaches. Table 3 describes the comparison among the suggested methods and some other methods reported in the literature $[2,9,10,30]$. Switches status, $P_{L}$, minimum and average voltage are reported in this table.

Table 3 illustrates that the loss $\left(P_{L T}\right)$ reduces while using BPSO by $31 \%$ from $152.20 \mathrm{~kW}$ to $104.87 \mathrm{~kW}$ and with CBPSO by $34 \%$ from $152.20 \mathrm{~kW}$ to $100.31 \mathrm{~kW}$. The minimum voltage $\left(V_{\min }\right)$ while using BPSO enhances from 0.9248 to $0.9489 \mathrm{p}$.u. and with CBPSO enhances from $0.9248 \mathrm{p}$. u. to 0.9505 p.u., while the average voltage enhances from 0.9553 to 0.9702 p.u. while using BPSO and from 0.9553 to 0.9712 p.u. with CBPSO. Thus, it is quite obvious from the comparison results that the CBPSO algorithm is achieved best results and it has high ability and superior in solving DNR problem than other methods. Figure 5 shows voltage profiles of the network while using BPSO and CBPSO. It is clear that the voltage at all nodes (except 19,20,21,22) were improves after reconfiguration. Finally, it is clear that from Figure 5 by using CBPSO greatly improves the voltage profile compared to using BPSO.

Table 3. Results of DNR for the IEEE 33-node power system at load factor $(\mu=0.875)$ while using BPSO, CBPSO and some other approaches

\begin{tabular}{ccccc}
\hline Approach & Open Switches & $P_{L T}(\mathrm{KW})$ & $V_{a v}(p . u)$. & $V_{\min }(p . u)$. \\
\hline Initial & sw33, sw34, sw35, sw36, sw37 & 152.20 & 0.9553 & 0.9248 \\
BPSO & sw7, sw9, sw13, sw32, sw37 & 104.87 & 0.9702 & 0.9489 \\
CBPSO & sw7, sw9, sw14, sw32, sw37 & 100.31 & 0.9712 & 0.9505 \\
FWA [2] & sw7, sw9, sw14, sw28, sw32 & 105.88 & 0.9716 & 0.9490 \\
MBFOA [9] & sw7, sw9, sw13, sw32, sw37 & 107.31 & 0.9720 & 0.9460 \\
ITS [10] & sw07, sw09, sw14, sw36, sw37 & 107.46 & 0.9698 & 0.9423 \\
SLR [30] & sw07, sw10, sw14, sw36, sw37 & 107.84 & 0.9697 & 0.9423 \\
\hline
\end{tabular}
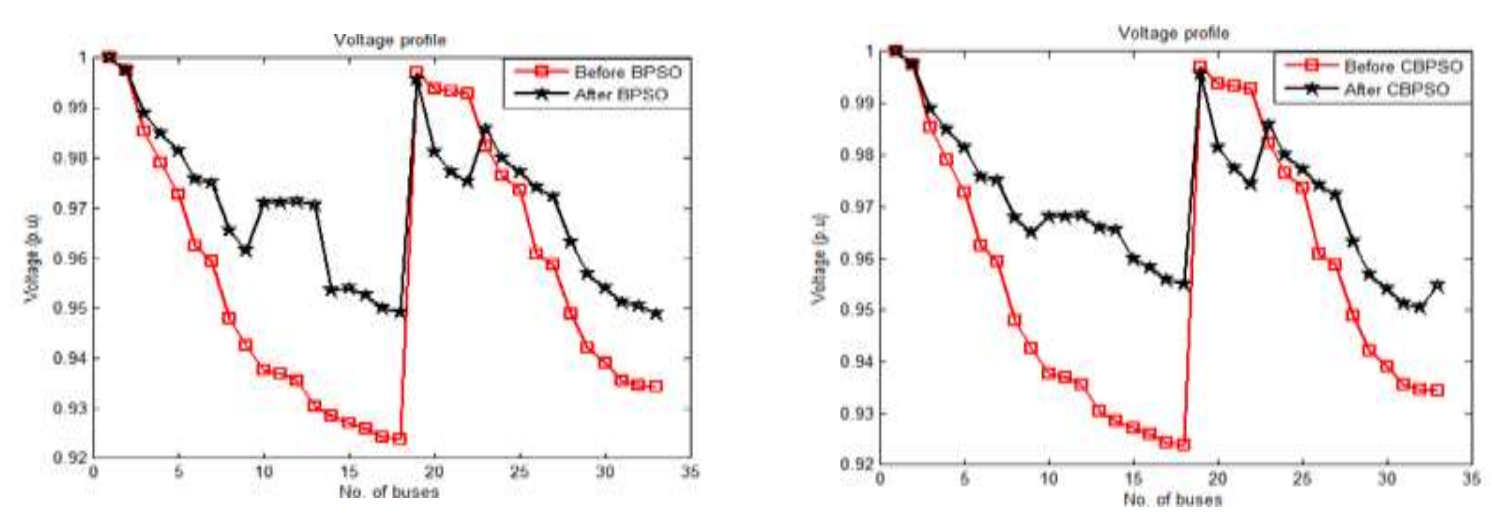

Figure 5. Voltage profile of DNR for the IEEE 33-node grid at load factor $(\mu=0.875)$ while using BPSO and CBPSO 


\subsubsection{At Loading with Variation Ratio $(\mu=1.125)$}

In this case, DNR is applied to load factor $(\mu=1.125)$ demand. IEEE 33-node is presented as test system for both the BPSO and WIBPSO approaches. Table 4 describes the comparison among the proposed methods and some other methods reported in the literature $[2,9,10,30]$. Switches status, $P_{L}$, minimum and average voltage are exposed in this table.

Table 4 indicates that the loss $\left(P_{L T}\right)$ reduces while using BPSO by $6 \%$ from $261.69 \mathrm{~kW}$ to $243.63 \mathrm{~kW}$ and with CBPSO by $34 \%$ from $261.69 \mathrm{~kW}$ to $171.08 \mathrm{~kW}$. The minimum voltage $\left(V_{\min }\right)$ while using BPSO enhances from 0.9011 to 0.9199 p. u. and with CBPSO enhances from 0.9011 to 0.9355 p.u., while the average voltage enhances from 0.9414 to 0.9482 p.u. while using BPSO and from 0.9414 to 0.9754 p.u. with CBPSO. So, it is quite obvious from the comparison results that the CBPSO algorithm is achieved best results and it has high ability and superior in solving DNR problem than other methods.

Figure 6 shows voltage profiles of the network while using BPSO and CBPSO. It is clear that the voltage at all nodes (except 19,20,21,22) were improves after reconfiguration. Finally, it is obvious that from Figure 6, the voltage profile by utilizing CBPSO is really improves compared to using BPSO.

Table 4. Results of DNR for the IEEE 33-node power system at load factor $(\mu=1.125)$ while using BPSO, CBPSO and some other approaches

\begin{tabular}{clccc}
\hline Approach & Open Switches & $P_{L T}(\mathrm{KW})$ & $V_{a v}(p . u)$. & $V_{\min }(p . u)$. \\
\hline Initial & sw33, sw34, sw35, sw36, sw37 & 261.69 & 0.9414 & 0.9011 \\
BPSO & sw7, sw9, sw13, sw32, sw37 & 243.63 & 0.9482 & 0.9199 \\
CBPSO & sw7, sw9, sw14, sw32, sw37 & 171.08 & 0.9754 & 0.9355 \\
FWA [2] & sw7, sw9, sw14, sw28, sw32 & 179.63 & 0.9631 & 0.9335 \\
MBFOA [9] & sw7, sw9, sw13, sw32, sw37 & 181.91 & 0.9636 \\
ITS [10] & sw07, sw09, sw14, sw36, sw37 & 182.29 & 0.9607 & 0.9295 \\
SLR [30] & sw07, sw10, sw14, sw36, sw37 & 182.95 & 0.9605 & 0.9247 \\
\hline
\end{tabular}
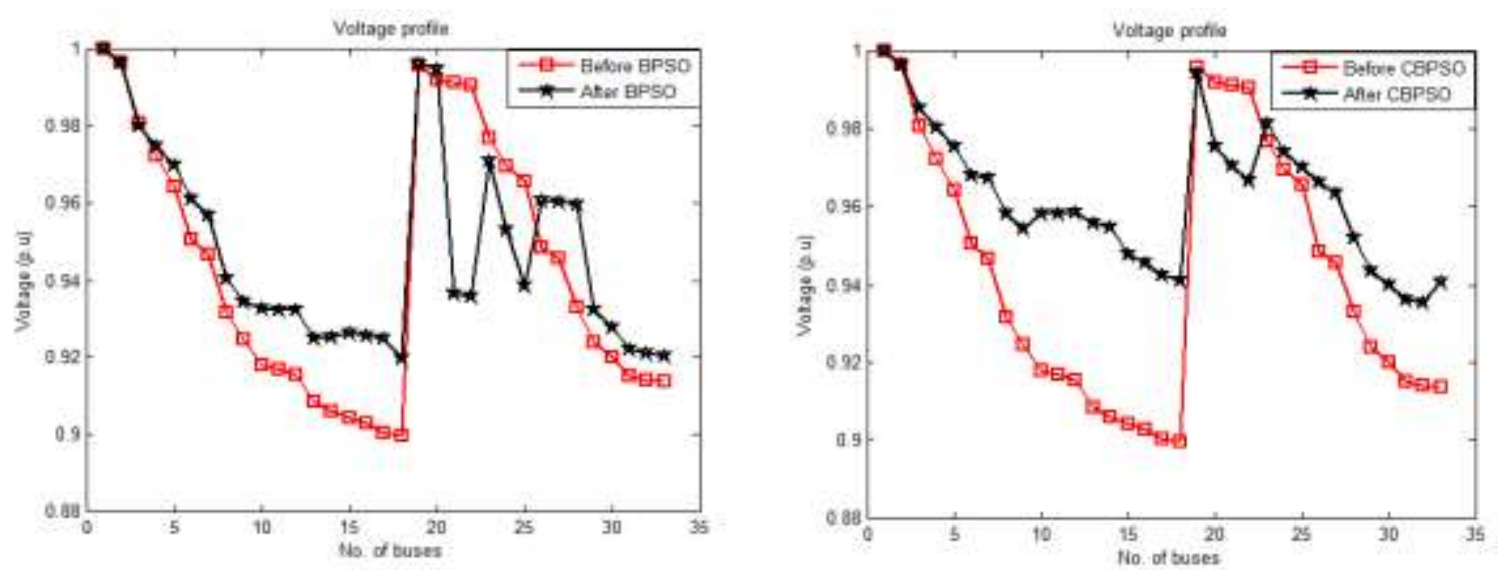

Figure 6. Voltage profile of DNR for the IEEE 33-node power system at load factor $(\mu=0.875)$ while using BPSO and CBPSO

\subsubsection{At Loading with Variation Ratio $(\mu=1.250)$}

In this case DNR is applied to the heavy load $(\mu=1.250)$ demand. IEEE 33-node is presented as a test system for both the BPSO and CBPSO approaches. Table 5 tabulates the comparison among the proposed methods and some other techniques mentioned in the literature $[2,9,10,30]$. Switches status, $P_{L}$, minimum and average voltage are given in Table 5.

Table 5 demonstrates that the loss $\left(P_{L T}\right)$ reduces while using BPSO by $6 \%$ from $329.85 \mathrm{~kW}$ to $305.81 \mathrm{~kW}$ and with CBPSO by $35 \%$ from $329.85 \mathrm{~kW}$ to $214.24 \mathrm{~kW}$. The minimum voltage $\left(V_{\min }\right)$ while using BPSO enhances from 0.8889 to 0.9102 p. u. and with CBPSO enhances from 0.8889 to 0.9279 p. u., while the average voltage enhances from 0.9342 to 0.9420 p.u. while using BPSO and from 0.9342 to 0.9581 p.u. with CBPSO. So, it is quite obvious from the comparison results that the CBPSO algorithm is achieved best results and it has high ability and superior in solving DNR problem than other methods.

Figure 7 shows voltage profiles of the network while using BPSO and CBPSO. It is clear that the voltage at all nodes (except 19,20,21,22) were improves after reconfiguration. Finally, it is clear that from Figure 7 by using CBPSO greatly improves the voltage profile compared to using BPSO. 
Table 5. Results of DNR for the IEEE 33-node power system at heavy load demands $(\mu=1.250)$ while using BPSO, CBPSO and some other approaches

\begin{tabular}{llccc}
\hline Approach & \multicolumn{1}{c}{ Open Switches } & $P_{L T}(\mathrm{KW})$ & $V_{a v}(p . u)$. & $V_{\min }(p . u)$. \\
\hline Initial & sw33, sw34, sw35, sw36, sw37 & 329.85 & 0.9342 & 0.8889 \\
BPSO & sw7, sw9, sw13, sw32, sw37 & 305.81 & 0.9420 & 0.9102 \\
CBPSO & sw7, sw9, sw14, sw32, sw37 & 214.24 & 0.9581 & 0.9279 \\
FWA [2] & sw7, sw9, sw14, sw28, sw32 & 224.25 & 0.9587 & 0.9256 \\
MBFOA [9] & sw7, sw9, sw13, sw32, sw37 & 227.52 & 0.9593 & 0.9211 \\
ITS [10] & sw07, sw09, sw14, sw36, sw37 & 228.08 & 0.9561 & 0.9156 \\
SLR [30] & sw07, sw10, sw14, sw36, sw37 & 228.92 & 0.9558 & 0.9156 \\
\hline
\end{tabular}
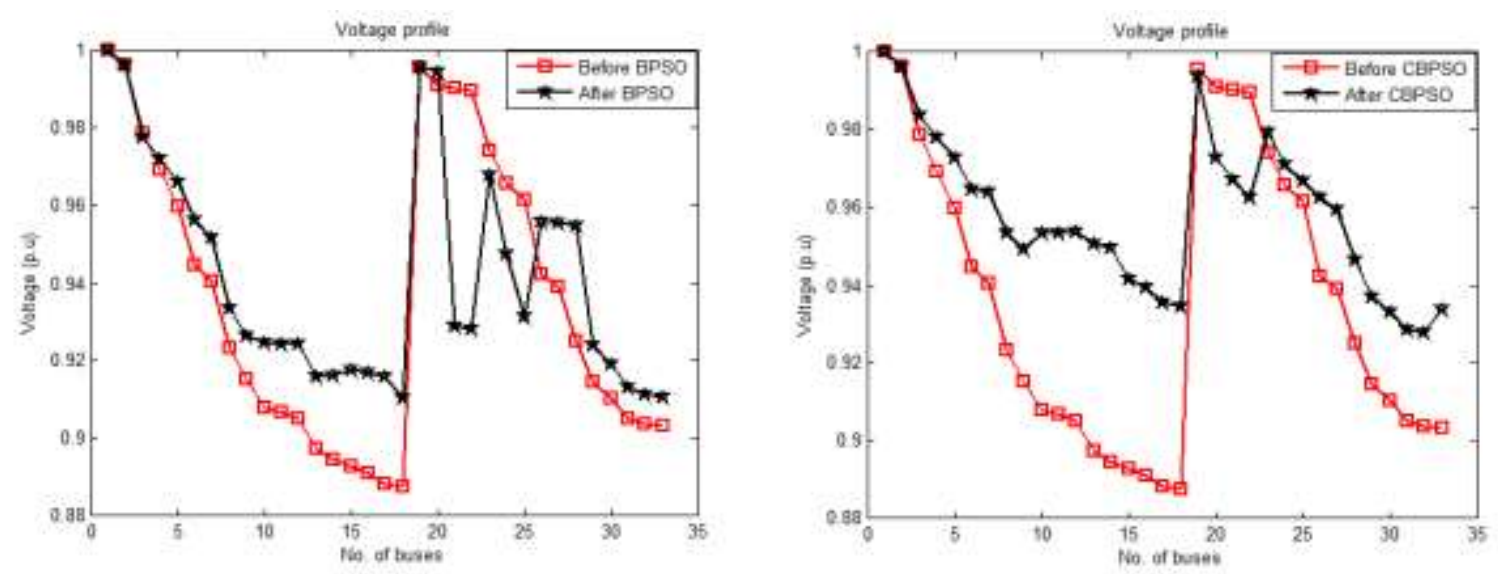

Figure 7. Voltage profile of DNR for the IEEE 33-node grid at heavy load demands $(\mu=1.250)$ while using BPSO and CBPSO

\section{CONCLUSION}

In this study, BPSO and Chaotic BPSO (CBPSO) algorithms are employed as a powerful optimization tools to find the optimal DNR problem. The main goals of utilizing the objective function are to decreasing active power loss $\left(\mathrm{P}_{\mathrm{L}}\right)$ and boosting voltage profile of grid. In this work, the chaotic strategy is merged with BPSO algorithm to form a strong hybrid algorithm called CBPSO algorithm to prevent the drawbacks of BPSO algorithm such as sticking in the local optima, slow convergence speed. Undeniably, the proposed CBPSO hybrid algorithm greatly helps to escape more easily from the local minima and thus, prevent the premature convergence due to the high ability and remarkable behaviour of the chaos theory than random search in BPSO technique. For testing these two algorithms, IEEE Node 33 system is utilized as a test system. Also, in this study employed two types of loads in order to test the proposed algorithms (i.e. BPSO and CBPSO algorithms) for solving this problem at any change in loads because the loads are variable from time to time and these two types of loads are: constant and variable loads. Simulation results prove that the CBPSO algorithm has best performance, biggest ability and high speed in convergence, also, it obtains lesser power loss with less number of iterations than BPSO and other reported algorithms. Also, the results indicate that CBPSO algorithm has high flexibility, effective and robustness for solving complex and non-linear problem in the power system, and it is believed that CBPSO will encouraging as a good candidate algorithm for future researchers.

\section{REFERENCES}

[1] Kalambe, S, Agnihotri, G. "Loss minimization techniques used in distribution network: bibliographical survey", Renew. Sustain. Energy Rev, vol. 29, pp. 184-200, 2014.

[2] Mohamed Imran, A.; Kowsalya, M. "A new power system reconfiguration scheme for power loss minimization and voltage profile enhancement using fireworks algorithm", Int. J. Electr. Power Energy Syst, vol. 62, pp. 312-322, 2014. 2014.

[3] E. Carpaneto, G. Chicco, and J. S. Akilimali, "Branch current decomposition method for loss allocation in radial distribution systems with distributed generation," IEEE Trans. Power Systems, vol. 21, no. 3, pp. 1170-1179, 2006.

[4] H. Kim, Y. ko, and K. H. Jung, "Artificial neural network-based feeder reconfiguration for loss reduction in distribution systems," IEEE Trans Power Delivery, vol. 8, no. 3, pp. 1356-1366, 1993.

[5] T. Taylor, and D. Lubkeman, "Implementation of heuristic search strategies for distribution feeder reconfiguration," IEEE Trans Power Delivery, vol. 5, no. 1, pp.239-246, 1990. 
[6] M. E. Baran, and F. F. Wu, "Network reconfiguration in distribution systems for loss reduction and load balancing," IEEE Trans. Power Delivery, vol. 4, no. 2, pp. 1401-1407, 1989.

[7] H. D. Chiang, and R.J Jumeau, "Optimal network reconfigurations in distribution systems: Part 1: A new formulation and a solution methodology," IEEE Trans. Power Delivery, vol. 5, no. 4, pp. 1902- 1909, 1990.

[8] G. K. V. Raju, and P.R. Bijwe, "Efficient reconfiguration of balanced and unbalanced distribution systems for loss minimization," IEE Proc. Gener. Transm. Distrib., vol. 2, no. 1, pp. 7-12, 2008.

[9] Naveen, S.; Sathish Kumar, K.; Rajalakshmi, K. "Distribution system reconfiguration for loss minimization using modified bacterial foraging optimization algorithm", Int. J. Electr. Power Energy Syst vol. 69, pp. 90-97, 2015.

[10] Nguyen, T.T.; Truong, A.V. "Distribution network reconfiguration for power loss minimization and voltage profile improvement using cuckoo search algorithm", Int. J. Electr. Power Energy Syst. vol. 68, pp. 233-242, 2015.

[11] Civanlar, S, Grainger, JJ, Yin, H, Lee, H: "Distribution feeder reconfiguration for loss reduction", IEEE T. Power Deliver, vol. 3, no. 3, pp.1217-1223, 1988.

[12] Broadwater, RP, Khan, AH, Shalaan, HE, Lee, RE, "Time varying load analysis to reduce distribution losses through reconfiguration", IEEE T. Power Deliver, vol. 8, no. 1, pp. 294-300, 1993.

[13] De Oliveira, L.W.; de Oliveira, E.J.; Gomes, F.V.; Silva, I.C.; Marcato, A.L.M.; Resende, P.V.C. "Artificial immune systems applied to the reconfiguration of electrical power distribution networks for energy loss minimization", Int. J. Electr. Power Energy Syst, vol. 56, pp. 64-74, 2014.

[14] Nara, K, Shiose, A, Kitagawoa, M, Ishihara, T, "Implementation of genetic algorithm for distribution systems loss minimum reconfiguration", IEEE Trans. Power Syst. vol. 7, pp. 1044-1051, 1992.

[15] Huang, Y-C, "Enhanced genetic algorithm-based fuzzy multi-objective approach to distribution network reconfiguration", Generation, Transmission and Distribution, IEE Proceedings vol. 149, no. 5, pp. 615-620, 2002.

[16] Su, C-T, Chung, CF, Chiou, J-P, "Distribution network reconfiguration for loss reduction by ant colony search algorithm", Electr. Pow. Syst. Res, vol. 75, pp. 190-199, 2005.

[17] Chiang, H-D, Rene, J-J, "Optimal network reconfiguration in distribution systems: part 1: a new formulation and a solution methodology", IEEE Trans. Power Deliv, vol. 5, pp. 1902-1908, 1990.

[18] Chiang, H-D, Rene, J-J, "Optimal network reconfiguration in distribution systems: part 2: solution algorithms and numerical results", IEEE Trans. Power Deliv, vol. 5, pp. 1568-1574, 1992.

[19] SrinivasaRao, R, Narasimham, SVL, RamalingaRaju, M, SrinivasaRao, A, "Optimal network reconfiguration of large-scale distribution system using harmony search algorithm", IEEE Trans. Power Syst. vol. 5, pp. 1080-1088 2011.

[20] Jin, X, Zhao, J, Sum, Y, Li, K, Zhang, B, "Distribution network reconfiguration for load balancing using Binary Particle Swarm optimization", International Conference on Power System Technology (POWERCON 2004), Singapore, pp. 507-510, 2004.

[21] C. T. Su, and C. S. Lee, "Network reconfiguration of distribution systems using improved mixed-integer hybrid differential evolution," IEEE Trans. Power Delivery, vol. 18, no. 3, pp. 1022-1027, 2003.

[22] Talha Ahmed Khan, Muhammad Alam, M. Junaid Tahir, Kushsairy Kadir, Zeeshan Shahid and M.S Mazliham, " Optimized health parameters using PSO: a cost-effective RFID based wearable gadget with less false alarm rate", Indonesian Journal of Electrical Engineering and Computer Science, vol. 15, no. 1, pp. 230-239, 2019.

[23] Ali Nasser Hussain, Ali Abdulabbas Abdullah, and Omar Muhammed Neda, "Modified Particle Swarm Optimization for Solution of Reactive Power Dispatch," Research Journal of Applied Sciences, Engineering and Technology, vol. 15, no. 8, pp. 316-327, 2018.

[24] Kanagasabai Lenin, "Power loss reduction by arctic wolf optimization algorithm," International Journal of Informatics and Communication Technology (IJ-ICT), vol. 8, no.3, pp. 3350-3357, 2019.

[25] R. C. Eberhart and J. Kennedy, "A discrete binary version of the particle swarm algorithm," Proc. of IEEE International Conference on Systems, Man, and Cybernetics, vol. 5, pp. 4104-4108, 1997.

[26] M.N. Dazahra, F. Elmariami, A. Belfqih, J. Boukherouaa, "Optimal Location of SVC using Particle Swarm Optimization and Voltage Stability Indexes," International Journal of Electrical and Computer Engineering (IJECE), vol. 6, no.6, pp. 2581-2588, 2016.

[27] Oladepo Olatunde and Hasimah Abdul Rahman, "Allocation of distributed generation and capacitor banks in distribution system," Indonesian Journal of Electrical Engineering and Computer Science, vol. 13, no. 2, pp. 437 447, 2019.

[28] M. Eslami, H. Shareef, A. Mohamed and M. Khajehzadeh, "Coordinated design of PSS and SVC damping controller using CPSO", in Proceeding of the 5th International Power Engineering and Optimization Conference (PEOCO2011), Shah Alam, Selangor, Malaysia, PP. 11-16, 2011.

[29] Ali Nasser Hussain, Ali Abdulabbas Abdullah and Omar Muhammed Neda, "Improvement of Reactive Power Dispatch by Using Hybrid Intelligent Optimization Technique Based on Chaotic and PSO Algorithm," International Journal of Applied Engineering Research, vol. 13, no. 6, pp. 3932-3938, 2018.

[30] Dall'Anese, E.; Giannakis, G.B, "Sparsity-Leveraging reconfiguration of smart distribution systems", IEEE Trans. Power Deliv. vol. 29, pp. 1417-1426, 2014. 